\title{
Unsupervised medical abortion with misoprostol among adolescent-what is the prospect of demedicalise abortion in Sub-Saharan Africa?
}

\author{
Adebiyi Gbadebo Adesiyun", Austin Ojabo²
}

${ }^{1}$ Department of Obstetrics \& Gynaecology, Ahmadu Bello University Teaching Hospital, Zaria, Nigeria;

${ }^{2}$ Department of Obstetrics and Gynaecology, Benue State University, Makurdi, Nigeria.

Email: biyi.adesiyun@yahoo.com

Received 20 October 2011; revised 21 November 2011; accepted 6 December 2011.

\section{ABSTRACT}

Objective: To find out clinical presentation and outcome of unsupervised use of misoprostol as abortifacent among adolescents presenting with abortion complications. Methods: Case series of thirty one adolescents that presented with abortion complications following unsupervised use of misoprostol. Results: Over a period of 3 years, 31 adolescents were seen, with median age of 17 years. Twenty nine (93.5\%) were unmarried and 22 (71\%) were in secondary school. Pregnancy duration was 3 months and above in $23(74.2 \%)$ of the patients. The cumulative dose of misoprostol tablet ingested was $2(400 \mu g)$ in 17 (54.8\%) of the patients. Twenty three (74.2\%) patients presented with incomplete abortion with mild sepsis while the remaining $8(25.8 \%)$ patients were admitted and managed with incomplete abortion with severe sepsis. Treatments offered were manual vacuum aspiration in $23(74.2 \%)$ patients, evacuation of retained product of conception under anaesthesia in 7 (22.6\%) patients and 1 (3.2\%) patient had laparotomy with uterine repair following inadvertent uterine perforation complicating curettage for incomplete abortion. Complications encountered were anaemia $67.7 \%$, uterine perforation $3.2 \%$, blood transfusion 9.7\% and diarrhoea in $8(25.8 \%)$ patients. Conclusion: Demedicalise abortion with misoprostol due to improper dosing protocol may be associated with incomplete abortion and its sequelae in an uninformed adolescent population. Establishment of adolescent $f$ riendly medical centre that offers post abortion care will go a long way in alleviating this problem.

Keywords: Demedicalise Abortion; Misoprostol; Adolescent; Medical Abortion

\section{INTRODUCTION}

The current generation of adolescents are more than a billion and they will be the largest generation in history making the transition from children to adulthood [1]. The transition period of adolescence is characterized by the onset of sexual relationships, marriage and child bearing. However, the biological and social impact of sexual activity in adolescence is associated with more conesquences in females than their male peers [1]. These consequences have been shown to conspicuously and untowardly shape the future of the female adolescent.

The changes in population dynamics resulting in migration to urban settlements and postponement of childbearing to allow for career attainment, has made pregnancy and childbearing at very early ages an unfavorable and an uncherish tradition contrary to what it was generations ago. The resultant delay in marriage coupled with low contraceptive prevalence typical of most developing countries, results in unwanted pregnancy [2]. It is estimated that nearly 70,000 women die annually from complications of unsafe abortion around the world. Over 69,000 of these deaths occur in developing countries while 23,000 occur in sub-Saharan African countries alone, representing an estimated 680 deaths per 100,000 abortion procedures in Africa [3].

Misoprostol, an orally active prostaglandin E1 analog was introduced into Nigeria medical practice for the management of peptic ulcer disease and lately for the prevention of postpartum haemorrhage. However, other countries have extended its use to some women problems like abortion, uterine evacuation and labour induction [4]. In Nigeria, the restrictive abortion law in place may encourage unsupervised use of misoprostol as an abortifacient. With this background, we studied adolescents presenting with complications of abortions following the use of self prescribed misoprostol in an at- 
tempt to induce an abortion.

\section{METHODS}

This was a descriptive observational case series of 31 adolescents that presented with complications of abortion following unsupervised use of misoprostol as an abortifacient. The patients were managed in Ahmadu Bello University Teaching Hospital, 345 Aeromedical Hospital and Alba Hospital, all located in Kaduna city, Northern Nigeria. The patients were seen between January 2005 and December 2008. All the patients obtained misoprostol medication without prescription. The cases were divided into 2 categories; mild and severe complications. Mild cases include those $\geq$ with temperature less than $100 \mathrm{~F}$ with absence of rigors, shock or generalized peritonitis. Severe cases are those with impending/early endotoxic shock, temperature greater than $100 \mathrm{~F}$ or subnormal, generalized peritonitis and evidence of bowel or bladder injury. For this study, anaemia was defined as hemoglobin level less than 10 grams per deciliter. The patients all had ultrasound examination to confirm incomplete abortion. Duration of pregnancy expressed in months was based on patient's estimation from last menstrual period.

The patients were either managed as out-patient or inpatient basis. Out-patient management was offered to those with mild cases and these include: uterine vacuum aspiration, oral antibiotic, analgesia, and oral haematinics for those with mild anaemia. Those with severe conditions were managed as in-patient. In-patient management included evacuation of retained product of conception under anaesthesia, intravenous fluids, parenteral antibiotics and blood transfusion depending on the severity of anaemia. Results are presented in numbers and percentages.

\section{RESULTS}

Over a period of 3 years, 31 patients with incomplete abortion following unsupervised use of misoprostol as abortifacient were seen. The median age was 17 years with age range of 14 to 19 years. Twenty nine $(93.5 \%)$ were single and $2(6.5 \%)$ are married. The 2 married patients were both less than 6 months into marriage. Twenty two $(71.0 \%)$ were secondary school students and $9(29.0 \%)$ were apprentice learning a vocation (Table 1).

Range of pregnancy duration was I to 6.5 months. Of the 31 patients, $17(54.8 \%)$ used an oral cumulative dose of 2 (400 microgram) misoprostol tablets, followed by 1 (200 microgram) tablet in $8(25.8 \%)$ patients and $3(600$ microgram) tablets in $6(19.4 \%)$ (Table 1). The peak duration of pregnancy before ingesting misoprostol was 3 months (17 patients, $54.8 \%$ ), followed by 4 months or above in $6(19.4 \%)$ patients (Figure 1).
Table 1. Profile of patients.

\begin{tabular}{ccc}
\hline VARIABLE & N = 31 & \% \\
\hline AGE (YEARS) & & \\
$<13$ & 0 & 0 \\
$14-16$ & 13 & 41.9 \\
$17-19$ & 18 & 58.1 \\
MARITAL STATUS & & \\
Single & 29 & 93.5 \\
Married & 2 & 6.5 \\
OCCUPATION & & \\
Secondary School & 22 & 71.0 \\
Apprentice & 9 & 29.0 \\
TOTAL DOSE OF MISOPROSTOL USED & & \\
1 tablet & 8 & 25.8 \\
2 tablets & 17 & 54.8 \\
3 tablets & 6 & 19.4 \\
\hline
\end{tabular}

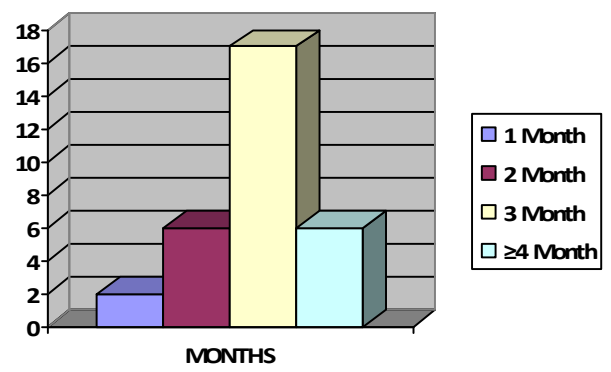

Figure 1. Bar chart of number of patients versus duration of pregnancy in months.

At presentation, based on the criteria, 23 (74.2\%) patients were classified to have mild incomplete septic abortion and $8(25.8 \%)$ patients with severe incomplete septic abortion (Table 2). None of the patients had been on antibiotics before presenting to the hospital. Time interval between ingestion of misoprostol to presentation in the hospital was between 10 and 20 days in $21(67.7 \%)$ patients and 30 to 40 days in $10(32.3 \%)$ patients (Table 2). In-patient management was offered to the entire 8 $(100 \%)$ patients with severe case and the 23 patients with mild case were managed as an out-patient.

Definitive treatment offered was uterine vacuum aspiration for $23(74.2 \%)$ patients, evacuation of retained product of conception under anaesthesia for $7(22.6 \%)$ patients and laparotomy/uterine repair for $1(3.2 \%)$ patient that had uterine perforation complicating uterine curettage done in an unsafe environment before presenting to the hospital. The patient that had laparotomy was a married secondary school student. Morbidities encountered were pyrexia $31(100 \%)$, anaemia 21 (67.7\%), uterine perforation complicating uterine curettage $1(3.2 \%)$ and diarrhoea in $8(25.8 \%)$ patients (Table 2). There was no mortality recorded. 
Table 2. Presentation, management and complications.

\begin{tabular}{ccc}
\hline Variable & N $=\mathbf{3 1}$ & $\mathbf{\%}$ \\
\hline $\begin{array}{c}\text { Mode of presentation } \\
\text { Mild incomplete septic abortion }\end{array}$ & 23 & 74.2 \\
Severe incomplete septic abortion & 8 & 25.8 \\
$\begin{array}{c}\text { Interval between ingestion of } \\
\text { misoprostol and presentation }\end{array}$ & & \\
10 - 20 days & 21 & 67.7 \\
30 - 40 days & 10 & 32.3 \\
TREATMENT & & \\
MVA & 23 & 74.2 \\
ERPC under anaesthesia & 7 & 22.6 \\
Laparotomy & 1 & 3.2 \\
MORBIDITY & & \\
Anaemia & 21 & 67.7 \\
Blood transfusion & 1 & 3.2 \\
Diarrhoea & 3 & 9.7 \\
Uterine perforation complicating curettage & 8 & 25.8 \\
\hline
\end{tabular}

MVA: Manual Vacuum Aspiration; ERPC: Evacuation of Retained Product of Conception.

\section{DISCUSSION}

Most developing countries like Nigeria have restrictive abortion law limiting access to safe abortion. Adolescents are more vulnerable to unwanted pregnancy and its complications because of secrecy surrounding sex at an early age due to peculiar socio-cultural background in most African Societies. Globally, medical abortion offers a new and better option to women seeking to terminate their pregnancy [5]. Medical methods of abortion hold the potential to improve women's health and advance their reproductive right [6]. With accessibility to medical abortion, women will no longer have to rely on surgical services considered to be less desirable and mostly unsafe in developing countries.

Supervision of medical abortion in a clinical setting is required at one stage or the other [1]; this makes the concept unfavorable for countries with restrictive abortion law. Supervision is paramount in low resource settings where majority are less educated and uninformed. All the patients managed in this study did not receive supervision before and/or after the use of misoprostol as abortifacient. Although authors have reported the possibility of "demedicalise abortion" that obligates medical supervision so long as easy access to medical care is available in case any problem arose [5-7]. However, for successful outcome of demedicalise abortion, women would need to accomplish the following; recognize that they are pregnant, estimate the duration of pregnancy, select appro- priate regimen, adhere to the correct protocol, manage adverse reactions and seek care for those that warrant medical attention, possibly notice and cope with expulsion of the embryo, and recognize a complete abortion [5]. Considering the aforementioned viz-a-viz the sociocultural and demographic characteristic obtainable in most African setting, it will be difficult to fulfill the criteria of demedicalise abortion in most resource constrained societies of Africa.

The peculiarities of the adolescent period especially in the developing countries where enormous stigma is associated with unwanted pregnancy, makes accomplishment of the above criteria almost impossible. Furthermore, the absence of adolescent friendly medical care has not helped the situation in most resource poor countries. Over a period of time, several regimens have been used for medical abortion. These include mifepristonegameprost, mifepristone-misoprostol and methotrexatemisoprostol [5]. However in Nigeria, out of all the drugs used for medical abortion, only misoprostol is easily accessible in terms of availability and cost. This attributes may have increasingly favored misoprostol as a commonly used abortifacient. Furthermore, lack of enforcement of drug prescription before dispensing policy in most developing countries is a major demerit towards the success of medical abortion .In this study, the pregnancies were either in the first or second trimester. In as much as authors [8-11] have documented the safety and efficacy of misoprostol alone in medical termination of $1^{\text {st }}$ and $2^{\text {nd }}$ trimester pregnancies. The route of administration and dosage regimen are of paramount importance. All the patients in this study used misoprostol orally. Oral route of administration have been associated with low efficacy, with success rates between 5\% and $11 \%$ in earlier studies $[12,13]$. On the contrary, vaginal route have success rates of between $89 \%$ and $94 \%$ $[8,10,11,14,15]$.

Treatment protocol is an important variable in the success rate of demedicalise abortion with misoprostol. Dose regimen ranging from $200 \mu \mathrm{g}$ to $800 \mu \mathrm{g}, 8$ to 12 hourly have been reported with good success [8,10,11,14, 15]. The adolescents in this series did not follow any particular regimen, which may be responsible for the low success rate and increase complication of incomplete abortion and its sequalae. Life table analysis estimating efficacy of misoprostol for first trimester pregnancy termination revealed the overall complete abortion rate of $88 \%$ to $93 \%$ with compliance to specific regime/protocol and intervention rate as low as 9\% [16]. The greatest percentage of deaths and serious morbidity resulting from unwanted pregnancies occur in developing countries [17]. In this series, the complication was mainly incomplete abortion and its sequalae like sepsis, anaemia and blood transfusion. Limitation of this study 
is that we did not rule out concomitant consumption of local herbal agents with abortifacient property.

Even though induced abortion is a criminal act in Nigeria, a lot of clandestine termination of pregnancy takes place on daily basis. Effort should be made towards setting up medical centers that are adolescent friendly, where counseling, routine medical assistance and care for abortion related complications can be rendered to patients. However, in other developing countries with unrestrictive abortion law, factors contributing to three tier delay in accessing health care must be considered in the success of demedicalise abortion [18].

\section{REFERENCES}

[1] The Alan Guttmacher Institute (1998) Into a new world -young women's sexual and reproductive lives: Young women in a changing world. New York, 5-9.

[2] Treffers, P.E., Olukoya, A.A., Ferguson, B.J. and Liljestrand, J. (2001) Care for adolescent pregnancy and childbirth. International Journal of Gynecology \& Obstetrics, 75, 111-121.

doi:10.1016/S0020-7292(01)00368-X

[3] Okonofua, F.E. (2004) Editorial: Breaking the silence on prevention of unsafe abortion in Africa. African Journal of Reproductive Health, 8, 7-8. doi:10.2307/3583174

[4] Population Council (2001) Misoprostol: An emerging technology for women's health, report of a seminar. In: Shannon, C.S. and Winikoff, B., Eds., New York, 2.

[5] Ellertson, C., Elul, B. and Winikoff, B. (1997) Can women use medical abortion without medical supervision? Reproductive Health Matters, 9, 149-161. doi:10.1016/S0968-8080(97)90019-7

[6] Elul, B. (1998) Medical methods of early abortion in developing countries. Consensus Statement, Contraception, 58, 257-259. doi:10.1016/S0010-7824(98)00109-7

[7] Winikoff, B., Siviel, Coyaji, K.J., et al. (1997) Safety efficacy and acceptability of medical abortion in China, Cuba and India: A comparative trial of mifepristonemisoprostol versus surgical abortion. American Journal of Obstetrics \& Gynecology, 176, 431-437. doi:10.1016/S0002-9378(97)70511-8

[8] Carabonell, J.L., Velazco, A., Varela, L., Tanda, R., Sanchez, C., Barambio, S., Chami, S., Valero, F.-A., Sanchez, S. and Mari, J. (2001) Misoprostol for abortion at 9 weeks - 12 weeks gestation in adolescents. European Journal of Contraception and Reproductive Healthcare, 6, 39-45. doi:10.1080/13625180008500367

[9] Carabonell, J.L., Varela, L., Velazco, A., Tanda, R., Barambio, S. and Chami, S. (2000) Vaginal misoprostol 600 microgram for early abortion. European Journal of Contraception and Reproductive Healthcare, 5, 46-51

[10] Herabutya, Y., Chanrachakul, B. and Punyavachira, P. (2001) Second trimester pregnancy termination: A comparison of 600 and 800 micrograms of intravaginal misoprostol. Journal of Obstetrics and Gynaecological Research, 27, 125-128. doi:10.1111/j.1447-0756.2001.tb01235.x

[11] Wong, K.S., Ngai, C.S., Yeo, E.L., Tang, L.C. and Ho, P.C. (2000) A comparism of two regimens of intravaginal misoporstol for termination of second trimester pregnancy: A randomized comparative trial. Human Reproduction, 15, 709-712. doi:10.1093/humrep/15.3.709

[12] Rabe, T., Basse, H., Thuro, H., Kiesel, L. and Runnebaum, B. (1987) Effect of the $\mathrm{PGE}_{1}$ methyl analog misoprostol on the pregnant uterus in the first trimester. $G e$ burtshilfe Frauenheilkd, 47, 324-331. doi:10.1055/s-2008-1035830

[13] Norman, J.E., Thong, K.J. and Baird, D.T. (1991) Uterine contractility and induction of abortion in early pregnancy by misoprostol and mifepristone. Lancet, 338, 1233-1236. doi:10.1016/0140-6736(91)92102-8

[14] Carabonell, J.L., Varela, L., Velazco, A. and Fernandez, C. (1997) The use of misoprostol for early termination of pregnancy. Contraception, 55, 165-168. doi:10.1016/S0010-7824(97)00020-6

[15] Carabonell, J.L., Varela, L., Velazco, A., Fernandez, C. and Sanchez, C. (1997) The use of misoprostol for abortion at $<$ or $=9$ weeks gestation. European Journal of Contraception and Reproductive Healthcare, 2, 181-185.

[16] Adjase, E.T. (2004) Misoprostol, an emerging technology for women's health, report of a seminar. In: Shannon, C.S. and Winikoff, B., Eds., Population Council, New York, 200, 5-12.

[17] World Health Organization (1994) Abortion: A tabulation of available data on the frequency and mortality of unsafe abortion. 2nd Edition, Geneva, Switzerland.

[18] Ibrahim, S.A., Iliyasu, Z. and Musa, J. (2006) Maternal mortality in jos university teaching hospital, Nigeria Trop Journal of Obstetrics and Gynaecology, 23, 152155 . 